\title{
2 次元成層せん断流における渦および吹き出しの基本解*1 Fundamental Solutions of Source and Vortex in a Two Dimensional Stratified Shear Flow
}

\author{
市川明夫*2 ・平 野 公 孝*3 \\ Akio ICHIKAWA, Kimitaka HIRANo \\ 菊 地 正 憲*3 \\ and Masanori KIKUCHI
}

Key Words: Fluid Dynamics, Fundamental Solution, Stratified Shear Flow, Lee Wave

\begin{abstract}
Fundamental solutions of a point source and a point vortex in a two-dimensional stratified shear flow are presented. The flow has the velocity increasing exponentially with $y$ and the density decreasing exponentially with $y$. The Euler equations are linearlized for small disturbances to the flow, and the method of Fourier transform is used to derive the solutions. Two types of solutions are obtained according to the magnitude of buoyancy effect; one is wavy form solution for large buoyancy and the other is non-wavy one for small buoyancy. For appropriate combination of source and vortex in a large buoyancy, the solution shows streamlines like lee waves.
\end{abstract}

\section{1. ま え がき}

近年. 電子計算機の発達に伴い, 流れの数值シミュ レーションが広く行われるようになってきた。この数 値シミュレーションは, 航空機や流体機械の, 設計初 期段階におけるパラメトリックスタディや，実験が困 難な現象の解析に，その役割を果たしている。

流れの数值シミュレーションの手法としては，大き く分けて, 差分法, 有限要素法, 特異点法(パネル法, または境界要素法）の三つがある。このうち，特異点 法では，流れに接する境界のみを対象に計算が行われ る.したがって，他の手法に比べて，末知数が格段に 少なくてすむほか，境界の形が複雑な場合や開領域の 扱いが容易であるという利点を持っている.

\footnotetext{
*1 平成 2 年 8 月 2 日原稿受理

*2 航空大学校

*3 宮崎大学工学部
}

この特異点法を用いる場合，解析すべき流れに対応 した，基本解 (Green 関数) が必要である。一様流に 対応した基本解は，古くから知られており"，それら を用いた特異点法によって，かなり複雑な形状をした 物体回りの流れの解析も行われている2).

一方，流体機械内部や地表付近の流れでは，主流は せん断を伴っているのが普通である．せん断流に対す る基本解については，非圧縮流に関して，その誘導や 詳しい検討がなされており゙゙，また，揚力面理論も試 みられている4) 要音速流に対しては，2 次元に関す る基本解が誘導され，それを用いた翼形特性の研究も 行われている5

このように, 特異点法の適用領域は広げられてきて いるが，解析対象として，海洋や高高度にわたる大気 を考えると，せん断流に加えて，密度成層の効果も考 虑に入れる必要がある。しかし，今までのところ、こ の成㬝せん断流を特異点法で扱った例はないように思 われる。

したがって, 本論では, 特異点法の適用領域を拡張 し，成層せん断流場をも扱えるようにする第一歩とし て, 次を行う

（1）特異点法による解析には，流れ場の基本解が 必要である.このため, 微少摄乱の仮定のもとに, 成 層せん断流中に固定された，単一の渦と吹き出しによ る擾乱速度や，摄乱圧力等の基本解を導くまた，そ れら摄乱源の持つ特異性を示す。

（2）基本解に関する若干の数値計算を行う。この ことによって, 流れのパラメータによって, 基本解の 様子が変わることが，直観的に示される。

なお，大規模な成層せん断流中の興味ある現象の一 つに，山越え気流の波動現象 (lee wave) がある6)。こ 
の現象は，差分法などの手法によって扱われてきてい るが、本研究の結果を応用すれば，特異点法による扱 いも可能となると思われる。

\section{主 な 記 号}

$C(x)$ : Fresnel 積分, (75) 式

$D: \hat{v}(y, \alpha)$ に関する特性方程式の判別式, (21) 式

$g:$ 重力加速度, $9.8 \mathrm{~m} / \mathrm{s}^{2}$

$I_{K 0}, I_{K 1}:$ 変形 Bessel 関数の半無限積分, (48), (49)式

$I_{N 0}, I_{N 1}$ ：第 2 種 Bessel 関数の半無限積分, $(50)$, (51) 式

$I_{\text {(1) }} \sim I_{(6)}:(69) \sim(74)$ 式

$K_{0}, K_{1}:$ 第 2 種変形 Bessel 関数

$m:$ 吹き出しの強さ

$N$ : 浮力振動数, (9) 式

$N_{0}, N_{1}$ : 第 2 種 Bessel 関数

$P(x, y), p(x, y):$ 圧力及び擾乱圧力

$R(x, y):$ 密度

$r:$ 原点からの距離, $r=\sqrt{x^{2}+y^{2}}$

$S(x)$ : Fresnel 積分, (75) 式

$U(x, y), u(x, y):$ 速度の $x$ 成分及び擾乱速度の $x$ 成 分

$V(x, y), v(x, y)$ : 速度の $y$ 成分及び擾乱速度の $y$ 成 分

$\tilde{v}(y, \alpha):$ Fourier 変換されたv

$x, y$ : 直交座標， $x$ は主流の方向， $y$ は主流に直 角で上方が正

$\alpha:$ Fourier 変換のパラメータ，(7)式

$\gamma:$ 渦の強さ

$\delta(x), \delta(y):$ Dirac のデルタ関数

$\kappa:$ 主流速度のせん断パラメータ，(19)式

$\lambda$ : 主流密度の成層パラメー夕，(19)式

$\mu: \mu^{2}=\lambda g / U_{0}^{2}-\lambda^{2} / 4-\kappa(\kappa-\lambda),(41)$ 式

$\nu: \nu^{2}=\lambda^{2} / 4+\kappa(\kappa-\lambda)-\lambda g / U_{0}^{2},(28)$ 式

$\zeta(x, y)$ : 擾乱渦度

$\rho(x, y):$ 擾乱密度

$\psi(x, y)$ : 摄乱流れ関数

添字等

( ) $)_{\infty}$ :主流での值

()$_{0}:$ 主流での， $y=0$ における值

()$^{R}:$ 正則部分

()$^{\prime}: x$ または $y$ に関する微分

\section{2. 線形化された基礎式}

座標系を第 1 図のようにとる。流れは，2次元，非 粘性，定常とする，すると，任意の点 $(x, y)$ におけ る速度 $(U, V)$, 圧力 $P$, 密度 $R$ は，主流の諸量と， 擾乱量との和として，次のように書くことができる．

$$
\left.\begin{array}{l}
U(x, y)=U_{\infty}(y)+u(x, y) \\
V(x, y)=v(x, y) \\
P(x, y)=p_{\infty}(y)+p(x, y) \\
R(x, y)=\rho_{\infty}(y)+\rho(x, y)
\end{array}\right\}
$$

なお， $p_{\infty}$ と $\rho_{\infty}$ は静水压平衡 $d p_{\infty} / d y=-\rho_{\infty} g$ を満 たしているとする。

摄乱量は，主流の諸量に比べて，小さいと仮定する と,

$$
\frac{u}{U_{\infty}}, \frac{v}{U_{\infty}}, \frac{p}{p_{\infty}}, \frac{\rho}{\rho_{\infty}} \ll 1
$$

原点に固定された束縛渦 $\gamma$ (以下，渦という) 及び. 吹き出し $m$ による，摄乱流れを支配する，線形化さ れた Euler 式及び連続の式は，

$$
\left.\begin{array}{l}
\rho_{\infty} U_{\infty} \frac{\partial u}{\partial x}+\rho_{\infty} U_{\infty}^{\prime} v=-\frac{\partial p}{\partial x} \\
\rho_{\infty} U_{\infty} \frac{\partial v}{\partial x}=-\frac{\partial p}{\partial y}-\rho g-\rho_{\infty} U_{\infty} \gamma \delta(x) \delta(y)
\end{array}\right\}
$$

であり、さらに流線に沿って, 密度は変化しないと仮 定すると、この線形化された表示式は，

$$
U_{\infty} \frac{\partial \rho}{\partial x}+\rho_{\infty}^{\prime} v=0
$$

となる。

(3)〜 (5) 式を組み合わせることによって，vに関 する, 次の形の線形化された、支配方程式が得られ

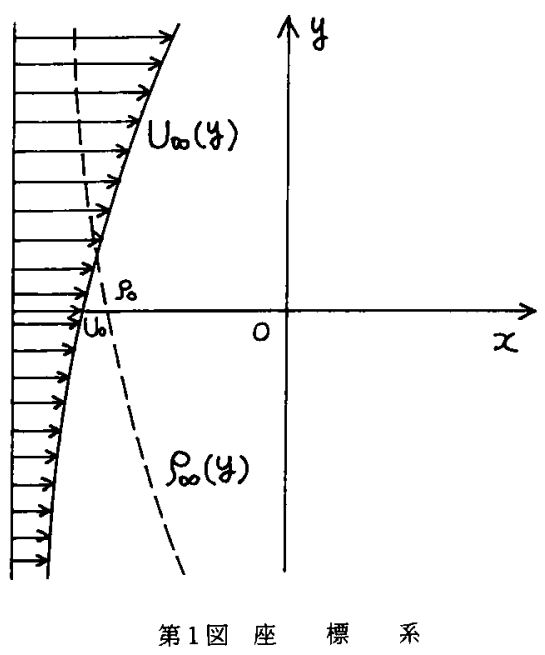


る。

$$
\begin{aligned}
\frac{\partial^{2} v}{\partial x^{2}} & +\frac{\partial^{2} v}{\partial y^{2}}+\frac{\rho_{\infty}^{\prime}}{\rho_{\infty}} \frac{\partial v}{\partial y}-\left[\frac{\left(\rho_{\infty} U_{\infty}^{\prime}\right)^{\prime}}{\rho_{\infty} U_{\infty}}+g \frac{\rho_{\infty}^{\prime}}{\rho_{\infty} U_{\infty}{ }^{2}}\right] v \\
= & -\gamma \delta^{\prime}(x) \delta(y)+m \delta(x)\left[\delta^{\prime}(y)\right. \\
& \left.+\frac{\left(\rho_{\infty} U_{\infty}\right)^{\prime}}{\rho_{\infty} U_{\infty}} \delta(y)\right]
\end{aligned}
$$

上式を偏微分方程式のままで解くことは困難である ので, 両辺をFourier 変換し, 変数分離をはかる。 $v(x, y)$ の Fourier 積分表示式は,

$$
v(x, y)=\frac{1}{2 \pi} \int_{-\infty}^{\infty} \bar{v}(x, \alpha) e^{i \alpha x} d \alpha
$$

であり, 同じく $\delta(x) も$ Fourier 積分表示式で表す. すると，(6)式は次となる。

$$
\begin{aligned}
\bar{v}^{\prime \prime} & +\frac{\rho_{\infty}^{\prime}}{\rho_{\infty}} \hat{v}^{\prime}-\left[\alpha^{2}+\frac{\left(\rho_{\infty} U_{\infty}^{\prime}\right)^{\prime}}{\rho_{\infty} U_{\infty}}-\frac{N^{2}}{U_{\infty}^{2}}\right] \bar{v} \\
& =-i \alpha \delta(y)+m\left[\delta^{\prime}(y)+\frac{\left(\rho_{\infty} U_{\infty}\right)^{\prime}}{\rho_{\infty} U_{\infty}} \delta(y)\right]
\end{aligned}
$$

以下, $\bar{v}(y, \alpha)=\bar{v}$ と略記し, 浮力振動数 $N$ は,

$$
N^{2}=-g \frac{\rho_{\infty}^{\prime}}{\rho_{\infty}}
$$

である。

(8)式の右辺は， $y \neq 0$ において0であり，よって (8)式は,

$$
\widehat{v}^{\prime \prime}+\frac{\rho_{\infty}^{\prime}}{\rho_{\infty}} \bar{v}^{\prime}-\left[\alpha^{2}+\frac{\left(\rho_{\infty} U_{\infty}^{\prime}\right)^{\prime}}{\rho_{\infty} U_{\infty}}-\frac{N^{2}}{U_{\infty}^{2}}\right] \bar{v}=0
$$

となる。

さらに, 渦または吹き出しの特異点の置かれた位置 $(y= \pm 0)$ ての条件は，（8)式の右辺の $\delta(y)$ の性質を 利用して, 次のように求められる.

(8)式を $y=-\varepsilon$ から $y=+\varepsilon$ まで積分をし， $\varepsilon \rightarrow 0$ とすると

$$
\begin{gathered}
{\left[\bar{v}^{\prime}(+0)-\hat{v}^{\prime}(-0)\right]+\frac{\rho_{\infty}^{\prime}}{\rho_{\infty}}(0)[\hat{v}(+0)-\hat{v}(-0)]} \\
=-i \alpha \gamma+m \frac{\left(\rho_{\infty} U_{\infty}\right)^{\prime}}{\rho_{\infty} U_{\infty}}(0)
\end{gathered}
$$

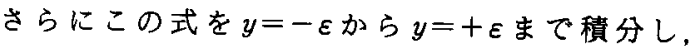
$\varepsilon \rightarrow 0$ とすると,

$$
\widehat{v}(+0)-\bar{v}(-0)=m
$$

これを(11)式に用いると，(11)式は，

$$
\widehat{v}^{\prime}(+0)-\widehat{v}^{\prime}(-0)=-i \alpha \gamma+m \frac{U_{\infty}^{\prime}}{U_{\infty}}(0)
$$

Fourier 変換された值 $\hat{v}(y, \alpha)$ に関する方程式 (10) 式が，流れ場を解析するための基礎式であり，(12)， （13）式が境界条件である。

これを解いて, 求まった $\hat{v}(y, \alpha)$ を( 7$)$ 式に代入す ることにより，v(x,y)を求めることができる.

また，（3）〜（５）式を用いることにより．摄乱速度 $u(x, y)$, 擾乱圧力 $p(x, y)$, 擾乱渦度 $\zeta(x, y)$, 擾乱
流れ関数 $\phi(x, y)$, 摄乱密度 $\rho(x, y)$ が $\hat{v}$ より，次の ように求めることができる.

$$
\begin{aligned}
& u(x, y)=\frac{i}{2 \pi} \int_{-\infty}^{\infty} \frac{1}{\alpha} \hat{v}^{\prime} e^{i a x} d \alpha \\
& \frac{p}{\rho_{\infty} U_{\infty}}(x, y)=-\frac{i}{2 \pi} \int_{-\infty}^{\infty} \frac{1}{\alpha}\left[\hat{v}^{\prime}-\frac{U_{\infty}^{\prime}}{U_{\infty}} v\right] e^{i \alpha x} d \alpha \\
& \zeta(x, y)=\frac{i}{2 \pi} \int_{-\infty}^{\infty}\left[\alpha \hat{v}-\frac{\bar{v}^{\prime \prime}}{\alpha}\right] e^{i a x} d \alpha \\
& \psi(x, y)=\frac{i}{2 \pi} \int_{-\infty}^{\infty} \frac{\bar{v}}{\alpha} e^{i a x} d \alpha \\
& \rho(x, y)=\frac{\rho_{\infty}^{\prime}}{U_{\infty}} \frac{i}{2 \pi} \int_{-\infty}^{\infty} \frac{\hat{v}}{\alpha} e^{i a x} d \alpha \\
& =\frac{\rho_{\infty}^{\prime}}{U_{\infty}} \psi(x, y)
\end{aligned}
$$

\section{3. 指数関数型成居せん断流の基本解}

3.1 指数関数型成層せん断流の基硞式 主流の速 度分布, 及び密度分布は, 次のように指数関数的に変 化すると仮定する。

$$
\left.\begin{array}{ll}
U_{\infty}(y)=U_{0} e^{\kappa y} & \kappa>0 \\
\rho_{\infty}(y)=\rho_{0} e^{-\lambda y} & \lambda>0
\end{array}\right\}
$$

(9)式より，浮力振動数 $N$ は定数となり, $N=$ $\sqrt{\lambda g}$ である.

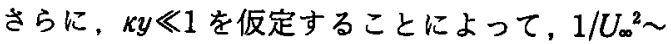
$1 / U_{0}^{2}$ が成立し，(10)式は次の定数係数を持つ，2 階 常微分方程式となる。

$$
\hat{v}^{\prime \prime}(y)-\lambda \hat{v}^{\prime}(y)-\left[\alpha^{2}+\kappa(\kappa-\lambda)-\frac{\lambda g}{U_{0}^{2}}\right] \hat{v}(y)=0
$$

上式が，解くべき基礎式である.

3.2 判別式による解の分類 基礎式(20)に対す る, 特性方程式の判別式 $D$ は, 次のようである.

$$
D=\alpha^{2}-\frac{\lambda g}{U_{0}^{2}}+\frac{\lambda^{2}}{4}+\kappa^{2}-\kappa \lambda
$$

上式の右辺第 1 項は，摃乱流れの波数を表し，第 2 項が浮力効果を表す. 第 $3 ， 4$ 項は, それぞれ，重力 の影響を除いた成層効果（以下単に成層効果という）， せん断流効果を表し，第 5 項はこれらの干渉効果を表 している。

基整式(20)の一般解は，判別式 $D$ の符号に従って, 次のように分類される.

$3.2 .1 \nu^{2}=\lambda^{2} / 4+x(x-\lambda)-\lambda g / U_{0}^{2}>0$ の場合 この場合は, 浮力効果が. 成層・せん断流効果より小 さいことを示し，波数 $\alpha$ のいかんにかかわらず $D>0$ であり，(20)式の一般解は，

$$
\hat{v}=C_{1} e^{\left(\frac{\lambda}{2}-\sqrt{D}\right)_{y}}+C_{2} e^{\left(\frac{\lambda}{2}+\sqrt{D}\right)_{y}}
$$

となる. 
$y \gtrless 0$ の各領域に関する解を， $y \rightarrow \pm \infty の$ 条件を考 虑して，次の形に仮定する。

$$
\left.\begin{array}{l}
y>0: \hat{v}=C_{1} e^{\left(\frac{\lambda}{2}-\sqrt{D}\right) y} \\
y<0: \bar{v}=C_{2} e^{\left(\frac{\lambda}{2}+\sqrt{D}\right)_{y}}
\end{array}\right\}
$$

境界条件 (12)，(13) 式を適用することにより， $C_{1}, C_{2}$ が定まり，その結果は次のようになる。

$$
\left.\begin{array}{rl}
y>0: \hat{v}= & {\left[\frac{\gamma i}{2} \frac{\alpha}{\sqrt{\alpha^{2}+\nu^{2}}}\right.} \\
& \left.+\frac{m}{2}\left(\frac{\lambda-2 \kappa}{2 \sqrt{\alpha^{2}+\nu^{2}}}+1\right)\right] e^{\left(\frac{\lambda}{2}-\sqrt{\alpha^{2}+\nu^{2}}\right) y} \\
y<0: \bar{v}= & {\left[\frac{\gamma i}{2} \frac{\alpha}{\sqrt{\alpha^{2}+\nu^{2}}}\right.} \\
& \left.+\frac{m}{2}\left(\frac{\lambda-2 \kappa}{2 \sqrt{\alpha^{2}+\nu^{2}}}-1\right)\right] e^{\left(\frac{\lambda}{2}+\sqrt{\alpha^{2}+\nu^{2}}\right) y}
\end{array}\right\}
$$

$3.2 .2 \mu^{2}=\lambda g / U_{0}^{2}-\lambda^{2} / 4-x(x-\lambda)>0$ 場 合 この場合は，浮力効果が，成層・せん断流効果より大 きいことを示し，波数 $\alpha$ により，次のように $D$ の符 号が変化する。

(1) $|a|>\mu: 3.2 .1$ と同しく $D>0$ であり， $\bar{v}$ は (24)式で与えられる.

(2) $|\alpha|<\mu ; D<0$ であり（20)式の一般解は,

$$
\tilde{v}=C_{1} e^{\left(\frac{\lambda}{2}-1,|D|\right)_{y}}+C_{2} e^{\left(\frac{\lambda}{2}+i, \mid D T\right) y}
$$

となる。

$y \gtrless 00$ 各領域に関する解を，波動の伝わる方向を 考慮し，次のように仮定する。

$$
\left.\begin{array}{l}
y>0: \hat{v}=C_{1} e^{\left(\frac{\lambda}{2}-\sqrt{\mu^{2}-\alpha^{2}} i\right) y} \\
y<0: \hat{v}=C_{2} e^{\left(\frac{\lambda}{2}+\sqrt{\mu^{2}-\alpha^{2}} i\right) y}
\end{array}\right\}
$$

境界条件(12)，(13)式によって， $C_{1}, C_{2}$ を定める と.

$$
\left.\begin{array}{rl}
y>0: \bar{v}= & {\left[\frac{\gamma}{2} \frac{\alpha}{\sqrt{\mu^{2}-\alpha^{2}}}\right.} \\
& \left.+\frac{m}{2}\left(1-\frac{i}{2} \frac{\lambda-2 \kappa}{\sqrt{\mu^{2}-\alpha^{2}}}\right)\right] e^{\left(\frac{\lambda}{2}-\sqrt{\mu^{2}-\alpha^{2}} i\right) y} \\
y<0: \bar{v}= & {\left[\frac{\gamma}{2} \frac{\alpha}{\sqrt{\mu^{2}-\alpha^{2}}}\right.} \\
& \left.-\frac{m}{2}\left(1+\frac{i}{2} \frac{\lambda-2 \kappa}{\sqrt{\mu^{2}-\alpha^{2}}}\right)\right] e^{\left(\frac{\lambda}{2}+\sqrt{\mu^{2}-\alpha^{2}}\right), y}
\end{array}\right\}
$$

となる。

3.3 浮力効果が小さい場合の基本解 浮力効果が 小さい場合，パラメータレは，次式で与えられる．

$$
\nu^{2}=\frac{\lambda^{2}}{4}+\kappa(\kappa-\lambda)-\frac{\lambda g}{U_{0}^{2}}>0
$$

(24)式を $y \gtrless 0 に つ い て ，$ 各々Fourier 逆変換を行 うと, $v$ は次となる。

$$
\begin{aligned}
& v(x, y)=-\frac{\gamma}{2 \pi} e^{\frac{d}{2} y} \frac{\nu x}{r} K_{1}(\nu r) \\
& +\frac{m}{2 \pi} e^{\frac{\lambda}{2} y}\left[\frac{\nu y}{r} K_{1}(\nu r)+\frac{\lambda-2 \kappa}{2} K_{0}(\nu r)\right]
\end{aligned}
$$

ただし， $r=\sqrt{x^{2}+y^{2}}$ であり， $K_{0}, K_{1}$ は第 2 種の変 形 Bessel 関数である.

同様にして，(14)〜(18)式を用いると，次のよう

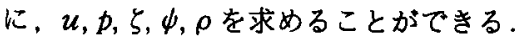

$$
\begin{aligned}
& u(x, y)=\frac{\gamma}{2 \pi} e^{\frac{\lambda}{2} y}\left[\nu \frac{y}{r} K_{1}(\nu r)-\frac{\lambda}{2} K_{n}(\nu r)\right] \\
& +\frac{m}{2 \pi} e^{\frac{\lambda}{2} y}\left[\nu \frac{x}{r} K_{1}(\nu r)\right. \\
& -\left\{\frac{\lambda g}{U_{0}^{2}}-\kappa\left(\kappa-\frac{\lambda}{2}\right)\right\} \int_{-\infty}^{x} K_{0}(\nu r) d x \\
& \left.-\kappa \nu y \int_{-\infty}^{x} \frac{1}{r} K_{1}(\nu r) d x\right] \\
& \frac{p}{\rho_{\infty} U_{\infty}}(x, y)=\frac{\gamma}{2 \pi} e^{\frac{\lambda}{2} y}\left[-\nu \frac{y}{r} K_{1}(\nu r)\right. \\
& \left.+\frac{\lambda-2 \kappa}{2} K_{0}(\nu r)\right] \\
& +\frac{m}{2 \pi} e^{\frac{\lambda}{2} \nu}\left[-\nu \frac{x}{r} K_{1}(\nu r)\right. \\
& \left.+\frac{\lambda g}{U_{0}^{2}} \int_{-\infty}^{x} K_{0}(\nu r) d x\right] \\
& \zeta(x, y)=\frac{\gamma}{2 \pi} e^{\frac{\lambda}{2} y}\left[-\lambda \nu \frac{y}{r} K_{1}(\nu r)\right. \\
& \left.+\left(\nu^{2}+\frac{\lambda^{2}}{4}\right) K_{0}(\nu r)\right] \\
& +\frac{m}{2 \pi} e^{\frac{\lambda}{2} y}\left[-\lambda \nu \frac{x}{r} K_{1}(\nu r)\right. \\
& -\nu\left(\frac{\lambda g}{U_{0}^{2}}-\kappa^{2}\right) \int_{-\infty}^{x} \frac{y}{r} K_{1}(\nu r) d x \\
& +\left\{\frac{\lambda}{2}\left[\frac{\lambda g}{U_{0}^{2}}-\kappa\left(\kappa-\frac{\lambda}{2}\right)\right]\right. \\
& \left.\left.-\kappa \nu^{2}\right\} \int_{-\infty}^{x} K_{0}(\nu r) d x\right] \\
& \phi(x, y)=\frac{\gamma}{2 \pi} e^{\frac{\lambda}{2} y\left[-K_{0}(\nu r)\right]} \\
& +\frac{m}{2 \pi} e^{\frac{\lambda}{2} y}\left[-\nu y \int_{-\infty}^{x} \frac{1}{r} K_{1}(\nu r) d x\right. \\
& \left.-\frac{\lambda-2 \kappa}{2} \int_{-\infty}^{x} K_{0}(\nu r) d x\right] \\
& \rho(x, y)=-\frac{\lambda \rho_{0}}{U_{0}} e^{-(\lambda+\kappa) y} \cdot \phi(x, y)
\end{aligned}
$$

これらの解は、いずれも第 2 種の変形 Bessel 関数 を含み， $r \rightarrow 0$ の特異性は次のようである.

$$
v \sim-\frac{\gamma}{2 \pi} \frac{x}{r^{2}}+\frac{m}{2 \pi}\left[\frac{y}{r^{2}}-\frac{\lambda-2 \kappa}{2} \ln r\right]
$$




$$
\begin{aligned}
& u \sim \frac{\gamma}{2 \pi}\left[\frac{y}{r^{2}}+\frac{\lambda}{2} \ln r\right]+\frac{m}{2 \pi} \frac{x}{r^{2}} \\
& \frac{p}{\rho_{\infty} U_{\infty}} \sim \frac{\gamma}{2 \pi}\left[-\frac{y}{r^{2}}-\frac{\lambda-2 \kappa}{2} \ln r\right]-\frac{m}{2 \pi} \frac{x}{r^{2}} \\
& \zeta \sim \frac{\gamma}{2 \pi}\left[-\frac{\lambda y}{r^{2}}-\left(\nu^{2}+\frac{\lambda^{2}}{4}\right) \ln r\right]-\frac{m}{2 \pi} \frac{\lambda x}{r^{2}} \\
& \psi \sim \frac{\gamma}{2 \pi} \ln r \\
& \rho \sim-\frac{\lambda \rho_{0}}{U_{0}} \frac{\gamma}{2 \pi} \ln r
\end{aligned}
$$

これらの特異性は，一様流中と同じ特異点に加え て，成層または，せん断流効果による特異性が加わっ たもの（ただし，(39)式を除く）となっている.

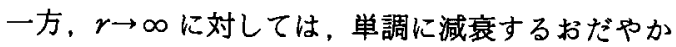
な解を持つ．ただし，線形の仮定により，yはある值 以下に制限される。

3.4 浮力効果が大きい場合の基本解 浮力効果が 大きい場合，パラメータ $\mu$ は，次式で与えられる。

$$
\mu^{2}=\frac{\lambda g}{U_{0}^{2}}-\frac{\lambda^{2}}{4}-\kappa(\kappa-\lambda)>0
$$

$\bar{v}$ の Fourier 逆変換は, $y \gtrless 0$ について,

$$
\begin{aligned}
v(x, y)= & \frac{1}{2 \pi}\left(\int_{-\infty}^{-\mu}+\int_{\mu}^{\infty}\right)[(24) \text { 式 }] e^{i \alpha x} d \alpha \\
& +\frac{1}{2 \pi} \int_{-\mu}^{\mu}[(27) \text { 式 }] e^{i a x} d \alpha
\end{aligned}
$$

であり，vは次の形となる。

$$
\begin{aligned}
v(x, y)= & \frac{\gamma}{2 \pi} e^{\frac{\lambda}{2} y}\left[\frac{\pi}{2} \mu \frac{x}{r} N_{1}(\mu r)\right] \\
& +\frac{m}{2 \pi} e^{\frac{\lambda}{2} y}\left[-\frac{\pi}{2} \mu \frac{y}{r} N_{1}(\mu r)\right. \\
& \left.-\frac{\lambda-2 \kappa}{2} \frac{\pi}{2} N_{0}(\mu r)\right]
\end{aligned}
$$

ただし， $r=\sqrt{x^{2}+y^{2}} て ゙ あ り ， N_{0}, N_{1}$ ，は第 2 種 Bessel 関数である.

さらに，(14)〜(18)式を用いることによって，u， $p, \zeta, \psi, \rho$ を次のように求めることができる.

$$
\begin{gathered}
u(x, y)=\frac{\gamma}{2 \pi} e^{\frac{\lambda}{2}}\left[-\frac{\pi}{2} \mu \frac{y}{r} N_{\mathrm{l}}(\mu r)+\frac{\lambda}{2} \frac{\pi}{2} N_{0}(\mu r)\right] \\
+\frac{m}{2 \pi} e^{\frac{\lambda}{2} y}\left[-\frac{\pi}{2} \mu \frac{x}{r} N_{1}(\mu r)\right. \\
+\left\{\frac{\lambda g}{U_{0}^{2}}-\kappa\left(\kappa-\frac{\lambda}{2}\right)\right\} \frac{\pi}{2} \int_{-\infty}^{x} N_{0}(\mu r) d x \\
\left.+\frac{\pi \kappa \mu y}{2} \int_{-\infty}^{x} \frac{1}{r} N_{1}(\mu r) d x\right] \\
\frac{p}{\rho_{\infty} U_{\infty}}(x, y)=\frac{\gamma}{2 \pi} e^{\frac{\lambda}{2} y}\left[\frac{\pi}{2} \mu \frac{y}{r} N_{1}(\mu r)\right. \\
\left.-\frac{\lambda-2 \kappa}{2} \frac{\pi}{2} N_{0}(\mu r)\right] \\
+\frac{m}{2 \pi} e^{\frac{\lambda}{2} y}\left[\frac{\pi}{2} \mu \frac{x}{r} N_{1}(\mu r)\right.
\end{gathered}
$$

$$
\begin{aligned}
& \left.-\frac{\lambda g}{U_{0}^{2}} \frac{\pi}{2} \int_{-\infty}^{x} N_{0}(\mu r) d x\right] \\
& \zeta(x, y)=\frac{\gamma}{2 \pi} e^{\frac{\lambda}{2} y}\left[\frac{\pi \lambda \mu}{2} \frac{y}{r} N_{1}(\mu r)\right. \\
& \left.-\frac{\pi}{2}\left(\frac{\lambda^{2}}{4}-\mu^{2}\right) N_{0}(\mu r)\right]
\end{aligned}
$$

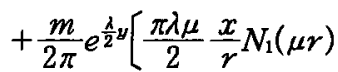

$$
\begin{aligned}
& +\frac{\pi}{2}\left(\frac{\lambda g}{U_{0}^{2}}-\kappa^{2}\right) \mu \int_{-\infty}^{x} \frac{y}{r} N_{1}(\mu r) d x \\
& -\frac{\pi}{2}\left[\frac{\lambda}{2}\left\{\frac{\lambda g}{U_{0}^{2}}-\kappa\left(\kappa-\frac{\lambda}{2}\right)\right\}\right. \\
& \left.\left.+\kappa \mu^{2}\right] \int_{-\infty}^{x} N_{0}(\mu r) d x\right] \\
& \psi(x, y)=\frac{\gamma}{2 \pi} e^{\frac{\lambda}{2} y}\left[\frac{\pi}{2} N_{0}(\mu r)\right] \\
& +\frac{m}{2 \pi} e^{\frac{\lambda}{2} y}\left[\frac{\pi \mu y}{2} \int_{-\infty}^{x} \frac{1}{r} N_{1}(\mu r) d x\right. \\
& \left.+\frac{\pi}{2} \frac{\lambda-2 \kappa}{2} \int_{-\infty}^{x} N_{0}(\mu r) d x\right] \\
& \rho(x, y)=-\frac{\lambda \rho_{0}}{\bar{U}_{0}} e^{-(\lambda+\kappa) y} \cdot \psi(x, y)
\end{aligned}
$$

これらの解は，第 2 種 Bessel 関数より成る. $r \rightarrow 0$ の特異性は, 浮力効果が小さい場合の特異性(35) (40)式と同一となり，一般のrに対しては，隇衰振 動的な解となる(ただし，yはある值に制限される).

\section{4. 数 值計算}

\subsection{Bessel 関数及び変形 Bessel 関数の半無限矰} 分の扱い 前項で得られた，(29)〜(34)式または (42)〜 (47) 式を数値計算することにより，摄乱速度や 擾乱圧力などを求めることができる.

上記の式に含まれる, Bessel 関数や変形 Bessel 関 数の值については, 高精度の近似式が容易に利用でき る.しかし, それらの半無限積分は, パラメータの值 によっては，特異積分となったり，あるいは，被積分 関数の収束性が悪化したりするので，改めて考察する 必要がある。

扱うべき半無限積分は次の四つである.

$$
\begin{aligned}
& I_{K 0}=\int_{-\infty}^{x} K_{0}(\nu r) d x, I_{K 1}=\int_{-\infty}^{x} \frac{1}{r} K_{1}(\nu r) d x \\
& I_{N 0}=\int_{-\infty}^{x} N_{0}(\mu r) d x, I_{N 1}=\int_{-\infty}^{x} \frac{1}{r} N_{1}(\mu r) d x,
\end{aligned}
$$

ただし， $r=\sqrt{x^{2}+y^{2}} て ゙ あ り ， \nu, \mu は(28)$ 式及び(41) 式で与えられる定数である。

\subsection{1 $I_{K 0}=\int_{-\infty}^{x} K_{0}(\nu r) d x$ の扱い}

(1) $y=0$ の場合 


$$
\int_{-\infty}^{0} K_{0}(\nu|x|) d x=\int_{0}^{\infty} K_{0}(\nu x) d x=\frac{\pi}{2 \nu}
$$

を用いて。

$$
I_{\kappa 0}=\frac{\pi}{2 \nu}+\int_{0}^{x} K_{0}(\nu|x|) d x
$$

上式第 2 項の被積分関数 $K_{0}$ は，積分範囲の下限で 特異となる.したがって，この積分は，特異項を差し 引いて行い，後に特異項を解析的に積分して加える， という操作を行う.その結果は，

$$
I_{K 0}=\frac{\pi}{2 \nu}+x(1-\ln \nu|x|)+\int_{0}^{x} K_{0}^{R}(\nu|x|) d x
$$
ただし，

$$
K_{0}^{R}(\nu|x|) \equiv K_{0}(\nu|x|)+\ln \nu|x|
$$

（2） $y \neq 0$ の場合

$$
I_{\kappa 0}=\frac{\pi}{2 \nu} e^{-\nu y}+\int_{0}^{x} K_{0}(\nu r) d x
$$

第 2 項は， $K_{0}$ の引数 $\nu r \neq 0$ であるので，問題なく 数値積分が可能である。

4.1.2 $I_{K 1}=\int_{-\infty}^{x} \frac{1}{r} K_{1}(\nu r) d x$ の扱い

(1) $y=0$ の場合

$$
\int_{0}^{\infty} \frac{1}{x} K_{1}(\nu x) d x=\lim _{y \rightarrow 0} \int_{0}^{\infty} \frac{1}{r} K_{1}(\nu r) d x=-\frac{\pi}{2}
$$

また， $K_{1}(\nu x)$ の $x \rightarrow 0$ での特異性を次のように分離 して書き。

$$
\begin{gathered}
K_{1}(\nu x)=K_{1}^{R}(\nu x)+\frac{1}{\nu x}+\frac{\nu x}{2} \ln \nu x, \\
K_{1}^{R}(\nu x)=0\left\{(\nu x)^{2}\right\}
\end{gathered}
$$

$\int_{0}^{x} \cdots d x$ の部分の積分を $(54)$ 式のようにして行うと, $I_{K 1}$ は次のようになる。

$$
\begin{aligned}
I_{K 1}= & -\frac{\pi}{2}-\frac{1}{\nu|x|}+\frac{\nu|x|}{2}(\ln \nu|x|-1) \\
& +\int_{0}^{x} \frac{1}{|x|} K_{1}^{R}(\nu|x|) d x
\end{aligned}
$$

被積分項は $0(x)$ であるので，問題はない。

（2） $y \neq 0$ の場合

$$
\int_{0}^{\infty} \frac{1}{r} K_{1}(\nu r) d x=\frac{\pi}{2 \nu y} e^{-\nu y}
$$

を用いて。

$$
I_{K 1}=\frac{\pi}{2 \nu y} e^{-\nu \nu}+\int_{0}^{x} \frac{1}{r} K_{1}(\nu r) d x
$$

第 2 項は $r \neq 0$ であるので, 問題はない

$$
\begin{aligned}
& \text { 4.1.3 } I_{N 0}=\int_{-\infty}^{x} N_{0}(\mu r) d x \text { の扱い } \\
& \text { (1) } y=0 \text { の場合 } \\
& I_{N 0}=\int_{0}^{\infty} N_{0}(\mu x) d x+\int_{0}^{x} N_{0}(\mu|x|) d x
\end{aligned}
$$

と同じ考えで行うと，結果は次のようになる．

$$
I_{N 0}=\frac{2 x}{\pi}(\ln \mu|x|-1)+\int_{0}^{x} N_{0}^{R}(\mu|x|) d x
$$

ただし

$$
N_{0}^{R}(\mu x)=N_{0}(\mu x)-2 / \pi \cdot \ln \mu x
$$

(2) $y \neq 0$ の場合

$$
I_{N 0}=\int_{0}^{\infty} N_{0}(\mu r) d x+\int_{0}^{x} N_{0}(\mu r) d x
$$

上式第 2 項は $r \rightarrow 0$ とはならないので, 直接に数值 積分可能である．問題は第 1 項の扱いである.

$N_{0}(x)$ の漸近形として，次の形を用いる ${ }^{7)}$.

$$
\begin{aligned}
N_{0}(x) & \sim \sqrt{\frac{2}{\pi x}}\left[\left(1-\frac{9}{128 x^{2}}\right) \sin \left(x-\frac{\pi}{4}\right)\right. \\
& \left.-\frac{1}{8 x} \cos \left(x-\frac{\pi}{4}\right)\right]
\end{aligned}
$$

すると，(65)式第 1 項は次のように書ける．

$$
\begin{aligned}
\int_{0}^{\infty} N_{0}(\mu r) d x= & \int_{0}^{\infty}\left\{N_{0}(\mu r)-\sqrt{\frac{2}{\pi \mu r}}[(1\right. \\
& \left.-\frac{9}{128 \mu^{2} r^{2}}\right) \sin \left(\mu r-\frac{\pi}{4}\right) \\
& \left.\left.-\frac{1}{8 \mu r} \cos \left(\mu r-\frac{\pi}{4}\right)\right]\right\} d x+I_{a d}^{0}
\end{aligned}
$$

ただし

$$
\begin{aligned}
I_{a d}^{0} \equiv & \sqrt{\frac{2}{\pi}} \int_{0}^{\infty}\left[\left(\frac{1}{\sqrt{\mu r}}-\frac{9}{128} \frac{1}{(\mu r)^{5 / 2}}\right) \sin (\mu r\right. \\
& \left.\left.-\frac{\pi}{4}\right)-\frac{1}{8(\mu r)^{3 / 2}} \cos \left(\mu r-\frac{\pi}{4}\right)\right] d x \\
= & \frac{1}{\sqrt{\pi}}\left[I_{(1)}-I_{(2)}-\frac{1}{8}\left(I_{(3)}+I_{(4)}\right)\right. \\
& \left.-\frac{9}{128}\left(I_{(5)}, I_{(6)}\right)\right]
\end{aligned}
$$

ここで(67)式右辺第 1 項は, 被積分関数が $x \rightarrow \infty$ に対してすみやかに 0 に近づくので，精度良く数值積 分が可能である.

また，(68)式の $I_{\text {【一 }} \sim I_{\text {(6) }}$ は次のように求められる. ただし， $\mu|y|=\eta$ とおく。

$$
\begin{aligned}
I_{\tilde{1},}= & \int_{0}^{\infty} \frac{\sin \mu r}{\sqrt{\mu r}} d x=\frac{1}{\mu} \int_{\eta}^{\infty} \sqrt{\frac{\tau}{\tau^{2}-\eta^{2}}} \sin \tau d \tau \\
= & \frac{1}{\mu} \int_{\eta}^{\infty}\left[\sqrt{\frac{\tau}{\tau^{2}-\eta^{2}}}-\frac{1}{\sqrt{\tau}}-\frac{\eta^{2}}{2} \frac{1}{\tau^{5 / 2}}\right. \\
& \left.-\frac{3 \eta^{4}}{8} \frac{1}{\tau^{9 / 2}}\right] \sin \tau d \tau \\
& +\frac{1}{\mu}\left[\frac{185 \sqrt{\eta}-12 \eta^{5 / 2}}{420} \sin \eta\right. \\
& +\frac{149 \eta^{3 / 2}-12 \eta^{7 / 2}}{210} \cos \eta+\left(1-\frac{2}{3} \eta^{2}\right. \\
& \left.\left.+\frac{2}{35} \eta^{4}\right)\left(\sqrt{\frac{\pi}{2}}-S(\eta)\right)\right]
\end{aligned}
$$

上式第 1 項は 0 である．また，第 2 項は (54) 式の扱い 


$$
\begin{aligned}
& I_{(2)}=\int_{0}^{\infty} \frac{\cos \mu r}{\sqrt{\mu r}} d x=\frac{1}{\mu} \int_{\eta}^{\infty} \sqrt{\frac{\tau}{\tau^{2}-\eta^{2}}} \cos \tau d \tau \\
& =\frac{1}{\mu} \int_{\eta}^{\infty}\left[\sqrt{\frac{\tau}{\tau^{2}-\eta^{2}}}-\frac{1}{\sqrt{\tau}}-\frac{\eta^{2}}{2} \frac{1}{\tau^{5 / 2}}\right. \\
& \left.-\frac{3 \eta^{4}}{8} \frac{1}{\tau^{9 / 2}}\right] \cos \tau d \tau \\
& +\frac{1}{\mu}\left[\frac{185 \sqrt{\eta}-12 \eta^{5 / 2}}{420} \cos \eta\right. \\
& -\frac{149 \eta^{3 / 2}-12 \eta^{7 / 2}}{210} \sin \eta \\
& \left.+\left(1-\frac{2}{3} \eta^{2}+\frac{2}{35} \eta^{4}\right)\left(\sqrt{\frac{\pi}{2}}-C(\eta)\right)\right] \\
& I_{3}=\int_{0}^{\infty} \frac{\sin \mu r}{(\mu r)^{3 / 2}} d x=\frac{1}{\mu} \int_{\eta}^{\infty} \frac{\sin \tau}{\sqrt{\tau} \sqrt{\tau^{2}-\eta^{2}}} d \tau \\
& =\frac{1}{\mu} \int_{\eta}^{\infty}\left[\frac{1}{\sqrt{\tau} \sqrt{\tau^{2}-\eta^{2}}}-\frac{1}{\tau^{3 / 2}}\right. \\
& \left.-\frac{\eta^{2}}{2} \frac{1}{\tau^{7 / 2}}\right] \sin \tau d \tau \\
& +\frac{1}{\mu}\left[\frac{33-4 \eta^{2}}{15 \sqrt{\eta}} \sin \eta+\frac{2 \sqrt{\eta}}{15} \cos \eta\right. \\
& \left.-\frac{4 \eta^{2}-30}{15}\left(\sqrt{\frac{\pi}{2}}-C(\eta)\right)\right] \\
& I_{(4)}=\int_{0}^{\infty} \frac{\cos \mu r}{(\mu r)^{3 / 2}} d x=\frac{1}{\mu} \int_{\eta}^{\infty} \frac{\cos \tau}{\sqrt{\tau} \sqrt{\tau^{2}-\eta^{2}}} d \tau \\
& =\frac{1}{\mu} \int_{\eta}^{\infty}\left[\frac{1}{\sqrt{\tau} \sqrt{\tau^{2}-\eta^{2}}}-\frac{1}{\tau^{3 / 2}}\right. \\
& \left.-\frac{\eta^{2}}{2} \frac{1}{\tau^{7 / 2}}\right] \cos \tau d \tau \\
& +\frac{1}{\mu}\left[\frac{33-4 \eta^{2}}{15 \sqrt{\eta}} \cos \eta-\frac{2 \sqrt{\eta}}{15} \sin \eta\right. \\
& \left.+\frac{4 \eta^{2}-30}{15}\left(\sqrt{\frac{\pi}{2}}-S(\eta)\right)\right] \\
& I_{(5)}=\int_{0}^{\infty} \frac{\sin \mu r}{(\mu r)^{5 / 2}} d x=\frac{1}{\mu} \int_{\eta}^{\infty} \frac{\sin \tau}{\tau^{3 / 2} \sqrt{\tau^{2}-\eta^{2}}} d \tau \\
& =\frac{1}{\mu} \int_{\eta}^{\infty}\left[\frac{1}{\tau^{3 / 2} \sqrt{\tau^{2}-\eta^{2}}}-\frac{1}{\tau^{5 / 2}}\right] \sin \tau d \tau \\
& +\frac{1}{\mu}\left[\frac{2}{3 \eta^{3 / 2}} \sin \eta+\frac{4}{3 \sqrt{\eta}} \cos \eta\right. \\
& \left.-\frac{4}{3}\left(\sqrt{\frac{\pi}{2}}-S(\eta)\right)\right] \\
& I_{(6)}=\int_{0}^{\infty} \frac{\cos \mu r}{(\mu r)^{5 / 2}} d x=\frac{1}{\mu} \int_{\eta}^{\infty} \frac{\cos \tau}{\tau^{3 / 2} \sqrt{\tau^{2}-\eta^{2}}} d \tau \\
& =\frac{1}{\mu} \int_{\eta}^{\infty}\left[\frac{1}{\tau^{3 / 2} \sqrt{\tau^{2}-\eta^{2}}}-\frac{1}{\tau^{5 / 2}}\right] \cos \tau d \tau \\
& +\frac{1}{\mu}\left[\frac{2}{3 \eta^{3 / 2}} \cos \eta-\frac{4}{3 \sqrt{\eta}} \sin \eta\right. \\
& \left.-\frac{4}{3}\left(\sqrt{\frac{\pi}{2}}-C(\eta)\right)\right]
\end{aligned}
$$

以上において，S,Cは次で定義される Fresnel 積 分であり。近似式によって容易に計算される。

$$
S(x)=\int_{0}^{x} \frac{\sin \tau}{\tau^{1 / 2}} d \tau, C(x)=\int_{0}^{x} \frac{\cos \tau}{\tau^{1 / 2}} d \tau
$$

(69)〜(74) 式によって計算される $I_{(1)} \sim I_{(6)}$ を(68)式 に用い，それをさらに(67)式に用いることによって (65)式第 1 項が計算される。

\subsection{4 $I_{N 1}=\int_{-\infty}^{x} \frac{1}{r} N_{\mathrm{l}}(\mu r) d x$ の扱い}

(1) $y=0$ の場合

$$
\begin{aligned}
I_{N 1} & =\int_{-\infty}^{x} \frac{1}{|x|} N_{1}(\mu|x|) d x \\
& =\int_{0}^{\infty} \frac{1}{x} N_{1}(\mu x) d x+\int_{0}^{x} \frac{1}{|x|} N_{1}(\mu|x|) d x
\end{aligned}
$$

上式右辺第 2 項は,

$$
\begin{aligned}
& N_{1}(\mu|x|)=N_{1}^{R}(\mu|x|)+\frac{\mu|x|}{\pi} \ln \mu|x|-\frac{2}{\pi \mu|x|}, \\
& N_{1}^{R}(\mu|x|)=0(|x|)
\end{aligned}
$$

と書いて，次のように計算される。

$$
\begin{aligned}
\int_{0}^{x} \frac{1}{|x|} N_{1}(\mu|x|) d x= & \int_{0}^{x} \frac{1}{|x|} N_{1}^{R}(\mu|x|) d x \\
& +\frac{\mu|x|}{\pi}(\ln \mu|x|-1)+\frac{2}{\pi \mu|x|}
\end{aligned}
$$

(76)式第 1 項は積分区間を, $0 \sim l, l \sim \infty$ の二つに 分け，前者の区間ては， $N_{1}(x)$ の原点近くの特異性を 考慮し，後者の区間ては， $N_{1}(x)$ の漸近形を考虑する ことにより，次のようになる。

$$
\begin{aligned}
& \int_{0}^{\infty} \frac{1}{x} N_{1}(\mu x) d x=I_{N 1}^{a}+I_{N 1}^{b} \\
& I_{N 1}^{a} \equiv \int_{0}^{l} \frac{1}{x} N_{1}(\mu x) d x \\
& \quad=\int_{0}^{l} \frac{N_{1}^{R}(\mu x)}{x} d x+\frac{\mu l}{\pi}(\ln \mu l-1)+\frac{2}{\pi \mu l}
\end{aligned}
$$

$N_{1}(x)$ の漸近形として, 次を用いで，

$$
N_{1}(x) \sim \sqrt{\frac{2}{\pi x}}\left\{\sin \left(x-\frac{3}{4} \pi\right)+\frac{3}{8 x} \cos \left(x-\frac{3}{4} \pi\right)\right\}
$$

すると，(79) 式右辺第 2 項は，

$$
\begin{aligned}
I_{N 1}^{b} \equiv & \int_{l}^{\infty} \frac{1}{x} N_{1}(\mu x) d x \\
= & \int_{l}^{\infty} \frac{1}{x}\left[N_{1}(\mu x)-\sqrt{\frac{2}{\pi \mu x}}\left\{\sin \left(\mu x-\frac{3}{4} \pi\right)\right.\right. \\
& \left.\left.+\frac{3}{8 \mu x} \cos \left(\mu x-\frac{3}{4} \pi\right)\right\}\right] d x \\
& -\frac{1}{2 \sqrt{\pi}}\left[\left(3+\frac{1}{2 \mu l}\right) \frac{\cos \mu l}{\sqrt{\mu l}}\right. \\
& \left.+\left(3-\frac{1}{2 \mu l}\right) \frac{\sin \mu l}{\sqrt{\mu l}}+3(S(\mu l)-C(\mu l))\right]
\end{aligned}
$$

以上(78)〜(82)式を(76) 式に用いることにより，y 
$=0$ の場合の $I_{N 1}$ が計算される.

(2) $y \neq 0$ の場合

$$
\begin{aligned}
I_{N 1} & =\int_{-\infty}^{x} \frac{1}{r} N_{1}(\mu r) d x \\
& =\int_{0}^{\infty} \frac{1}{r} N_{1}(\mu r) d x+\int_{0}^{x} \frac{1}{r} N_{1}(\mu r) d x
\end{aligned}
$$

上式第 2 項は $r \rightarrow 0$ とはならないので，直接に数値 槽分可能である.

上式第 1 項は， $N_{1}(x)$ の渐近形として，再び(81) 式 の形を用い, $\cos \mu r /(\mu r)^{\frac{5}{2}}, \sin \mu r /(\mu r)^{\frac{5}{2}}$ の積分を。 4.1.3 項と同様な扱いをすることによって，次の形と なる。

$$
\begin{aligned}
& \int_{0}^{\infty} \frac{1}{r} N_{1}(\mu r) d x= \int_{0}^{\infty} \frac{1}{r}\left[N_{1}(\mu r)\right. \\
&-\sqrt{\frac{2}{\pi \mu r}}\left\{\sin \left(\mu r-\frac{3}{4} \pi\right)\right. \\
&\left.\left.+\frac{3}{8 \mu r} \cos \left(\mu r-\frac{3}{4} \pi\right)\right\}\right] d x \\
&+I_{a d}^{1} \\
& I_{a d}^{1} \equiv \sqrt{\frac{2}{\pi \mu}} \int_{0}^{\infty} \frac{1}{r^{3 / 2}}\left\{\sin \left(\mu r-\frac{3}{4} \pi\right)\right. \\
&\left.+\frac{3}{8 \mu r} \cos \left(\mu r-\frac{3}{4} \pi\right)\right\} d x \\
&=-\frac{\mu}{\sqrt{\pi}}\left[I_{(3)}+I_{(4)}-\frac{3}{8}\left(I_{(5)}-I_{(6)}\right)\right]
\end{aligned}
$$

$I_{(3)}, I_{(4) ~}$ (71)，(72)式で与えられるものであり． $I_{5}, I_{(6)}$ (73)，(74)式でちえられるものである.

以上(84)，(85)式及び，(71)〜 (74) 式を(83)式に用 いることによって, $y \neq 0$ の場合の $I_{N 1}$ を求めること ができる。

4.2 若干の計算例 浮力効果が小さい場合 $\left(\nu^{2}>\right.$ $0)$ 及び，浮力効果が大きい場合 $\left(-\nu^{2}=\mu^{2}>0\right) k つ い$ て、若干の計算結果を示す。

計算パラメータのスケールは任意にとることができ るが、ここでは大気のスケールを意識し，長さの単位 を $\mathrm{km}$ ととる.したがって, 重力加速度の值は $g=$

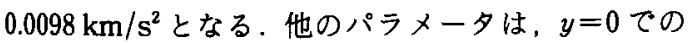
主流速度 $U_{0}=0.02 \mathrm{~km} / \mathrm{s}$, 主流速度のせん断パラメー 夕 $k=0.1 / \mathrm{km}$ とし，成層のパラメータは $\lambda=0.1 / \mathrm{km}$ (成層が著しい場合)及び， $\lambda=0.0001 / \mathrm{km}$ (成層がわず かな場合）ととる，また，吹き出しと渦の強さは， $m$ $=0.02 \mathrm{~km}^{2} / \mathrm{s}$ 及 $\mho m=0 \mathrm{~km}^{2} / \mathrm{s}, \gamma=-0.02 \mathrm{~km}^{2} / \mathrm{s}$ 及 ひ $\gamma=0 \mathrm{~km}^{2} / \mathrm{s}$ ととる.

第 2 図〜第 6 図は，浮力効果が小さい場合の結果て ある. 上記の $g, U_{0}, k$ に対しては， $\lambda<0.000406$ に対 して $\nu^{2}>0$ となり，振動的でない結果が得られる。同 図住 $\lambda=0.0001$ と与えた結果であるが. $\lambda=0 て ゙ も \lambda=$ 0.0004 でも結果はあまり変わらない。

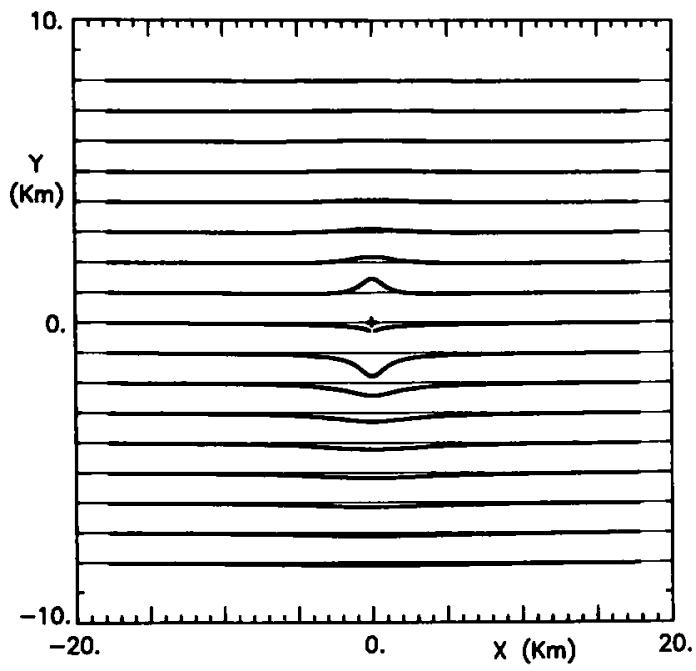

第 2 図真速度 $v$ の分布 $\left(U_{0}=0.02 \mathrm{~km} / \mathrm{s}, k=0.1 / \mathrm{km}\right.$, $\lambda=0.0001 / \mathrm{km}, m=0.02 \mathrm{~km}^{2} / \mathrm{s}, \gamma=0 \mathrm{~km}^{2} / \mathrm{s}$ )

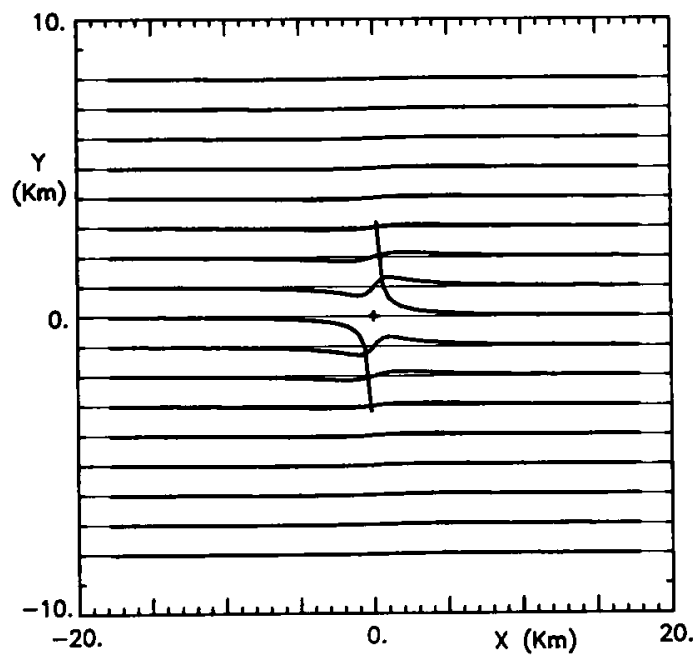

第 3 図垂直速度 $v$ の分布 $\left(U_{0}=0.02 \mathrm{~km} / \mathrm{s}, \kappa=0.1 / \mathrm{km}\right.$, $\left.\lambda=0.0001 / \mathrm{km}, m=0 \mathrm{~km}^{2} / \mathrm{s}, \gamma=-0.02 \mathrm{~km}^{2} / \mathrm{s}\right)$

第 2 図一第 4 図は，各々吹き出しのみ与えたもの。 渦のみ与えたもの，吹き出しと渦の両方与えたもの の. 垂直速度成分 $v$ の分布である. 中心 $(x=0, y=$ 0)に吹き出し，または渦が㧍かれ，周囲の 1 目盛り は $1 \mathrm{~km}$ を表す。また，vを示す曲線にほほ重なって いる細線は，vの基準を示している. 即ち, 細線より 上に出た部分では $v か ゙$ 正，下に出た部分では $v$ の負 を表している。なお，vの大きさは，目盛りに対して 200 倍されている。

これらの図の $v$ の分布は, 直感と合う結果を与え 


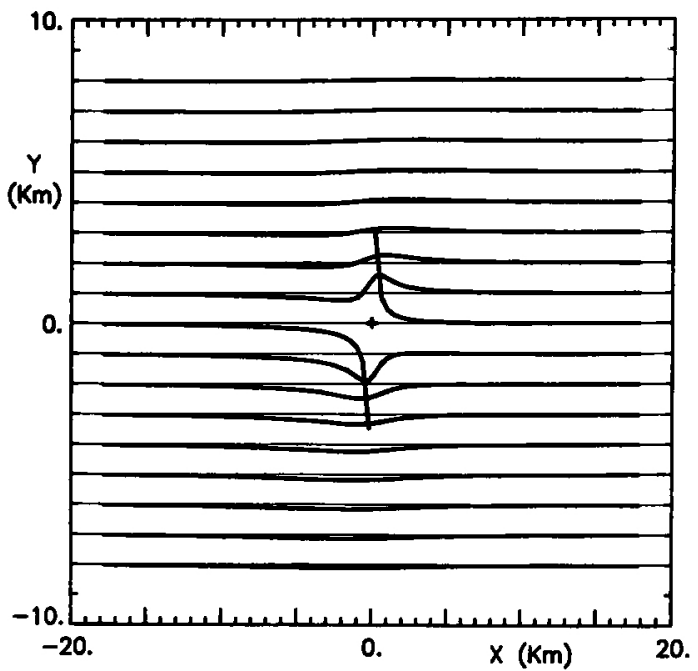

第 4 図垂直速度 $v$ の分布 $\left(U_{0}=0.02 \mathrm{~km} / \mathrm{s}, \kappa=0.1 / \mathrm{km}\right.$, $\lambda=0.0001 / \mathrm{km}, \quad m=0.02 \mathrm{~km}^{2} / \mathrm{s}, \quad \gamma=-0.02$ $\mathrm{km}^{2} / \mathrm{s}$ )

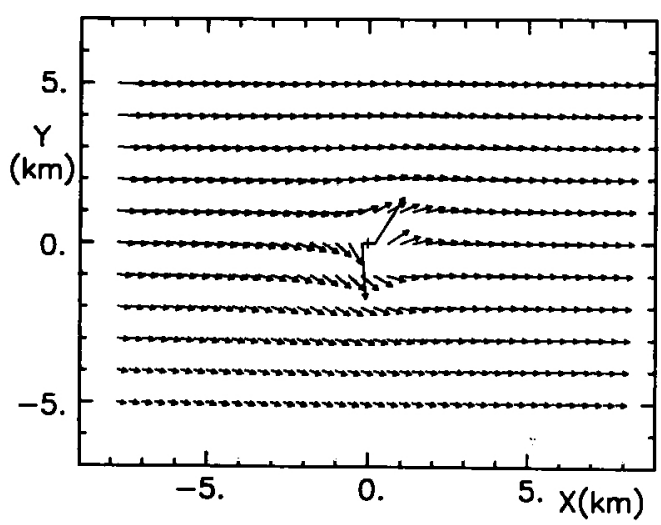

第 5 図流速べクトルの分布 $\left(U_{0}=0.02 \mathrm{~km} / \mathrm{s}, \kappa=\right.$ $0.1 / \mathrm{km}, \lambda=0.0001 / \mathrm{km}, m=0.02 \mathrm{~km}^{2} / \mathrm{s}, \gamma=$ $-0.02 \mathrm{~km}^{2} / \mathrm{s}$ )

ており，また，渦及び吹き出しの特異点の影響は，比 較的特異点近傍に限られているのがわかる。

第 5 図は，第 4 図と同じ条件の結果を，流速べクト ルで示したものである(原点付近のみ)．流速の大きさ は，目盛りに対して， $x$ 方向が 25 倍, $y$ 方向が 100 倍されている．したがって，流速へクトルの傾きは， 4 倍に誇張されている。

これら流速べトルも，特異点近くで，渦の影䪪 (反時計方向回転)及び吹き出しの影輩を示しているこ とが、よくわかる。

第 6 図は，第 4,5 図と同じ条件での流線を示した ものである．流線が上方で密となっているのは，上方 ての流速が大きいことに対応している，また，原点に

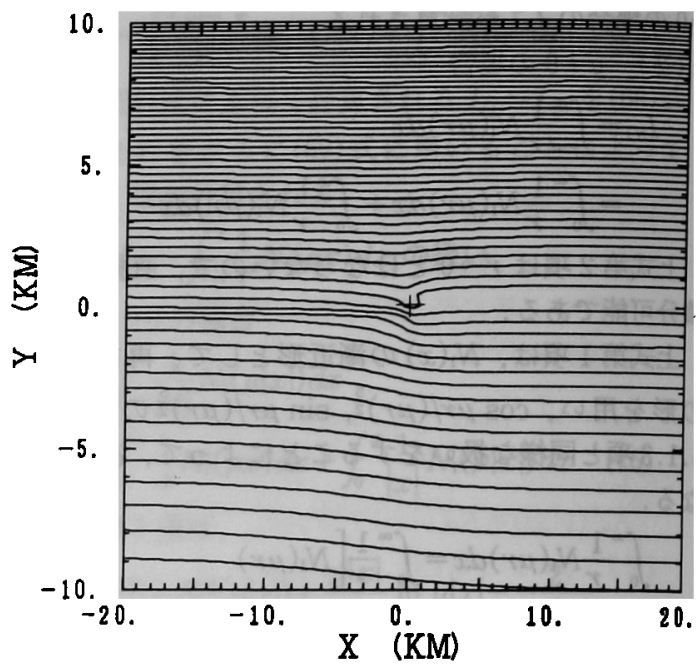

第 6 図流線図 $\left(U_{0}=0.02 \mathrm{~km} / \mathrm{s}, \kappa=0.1 / \mathrm{km}, \lambda=\right.$ $0.0001 / \mathrm{km}, m=0.02 \mathrm{~km}^{2} / \mathrm{s}, \gamma=-0.02 \mathrm{~km}^{2} / \mathrm{s}$ )

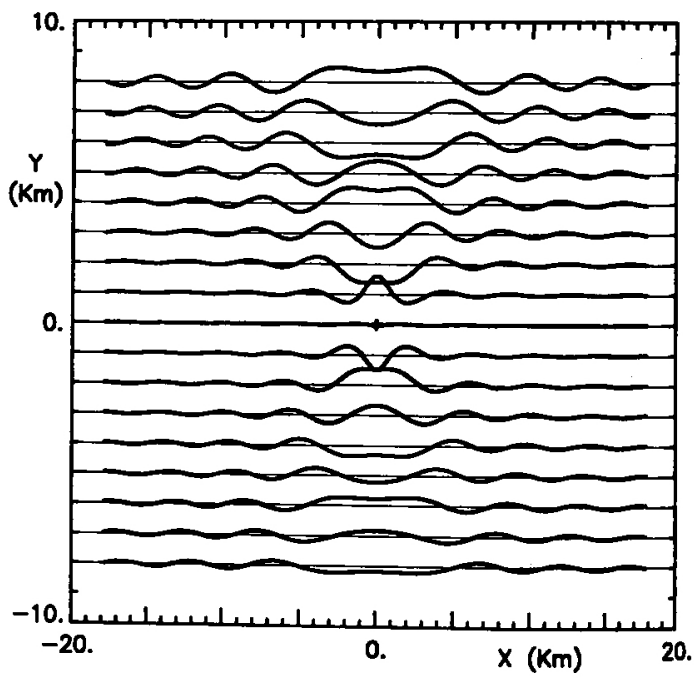

第 7 図垂直速度 $v$ の分布 $\left(U_{0}=0.02 \mathrm{~km} / \mathrm{s}, k=0.1 / \mathrm{km}\right.$, $\left.\lambda=0.1 / \mathrm{km}, m=0.02 \mathrm{~km}^{2} / \mathrm{s}, \quad \gamma=0 \mathrm{~km}^{2} / \mathrm{s}\right)$

おかれた特異性の影整は，原点より遠ざるにつれて 小さくなっているが，その小さくなり方は，上方でよ りすみやかである，ということがかかる，

第 7 図一第 11 図は，浮力効果が大きい場合の結果 であり， $\lambda=0.1$ と与えられている。この入は，地球 上での等温大気を考えた場合のスケールハイト $H$ と $\lambda=1 / H$ の関係にあり, 実際の場合 $(H \fallingdotseq 8 \mathrm{~km}, \lambda=$ $0.12 / \mathrm{km})$ に近い值()である。

第 7 図〜第 9 図は，各々吹き出し，渦，及び吹き" しと渦を中心においたときの，vの分布を表している （vの大きさは，目盛りに対して200倍されいろ 


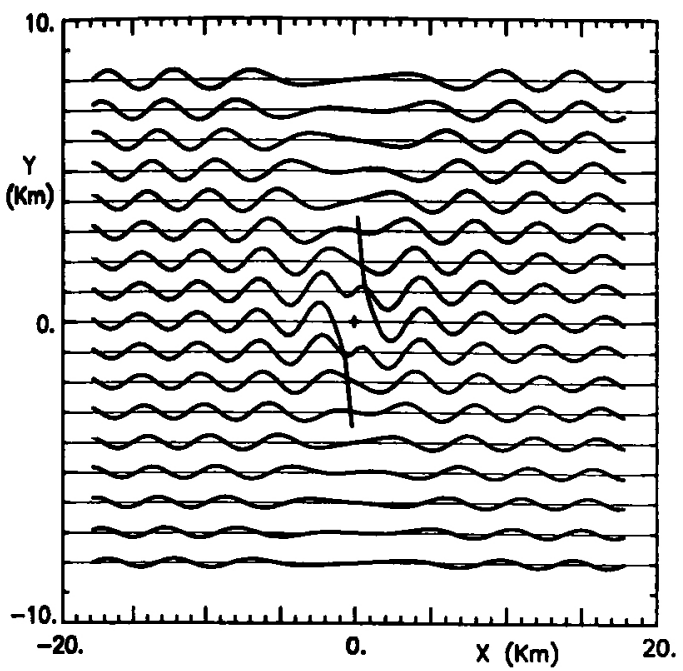

第 8 図垂直速度 $v$ 分布 $\left(U_{0}=0.02 \mathrm{~km} / \mathrm{s}, \kappa=0.1 / \mathrm{km}\right.$, $\left.\lambda=0.1 / \mathrm{km}, m=0 \mathrm{~km}^{2} / \mathrm{s}, \gamma=-0.02 \mathrm{~km}^{2} / \mathrm{s}\right)$

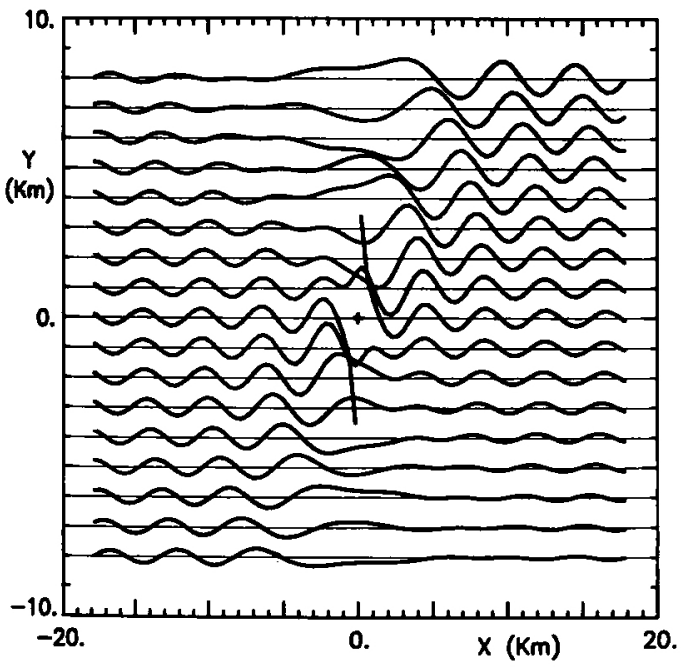

第 9 図草速度 $v$ の分布 $\left(U_{0}=0.02 \mathrm{~km} / \mathrm{s}, k=0.1 / \mathrm{km}\right.$, $\lambda=0.1 / \mathrm{km}, m=0.02 \mathrm{~km}^{2} / \mathrm{s}, \gamma=-0.02 \mathrm{~km}^{2} / \mathrm{s}$ )

これらの図において、いずれもvが振動的になって おり，かつ，特異点の遠方まて影響が及んているのが わかる．特に第 9 図は，重ね合わせる吹き出しと渦の 符号の組合せを適当に選べは，特異点の上方下流（及 ひ下方上流）に, lee wave 状の波動を作り出せること を示している。したがって,$y=0$ 上に地面及び山が ある場合，そこでの境界条件を満たすように，適当な 強さの吹き出し，及び渦を分布させることによって， 山越え気流の lee wave が計算できるのではないかと， 考えられる。

第 10 図は，第 9 図と同じものを，流速ベクトルで

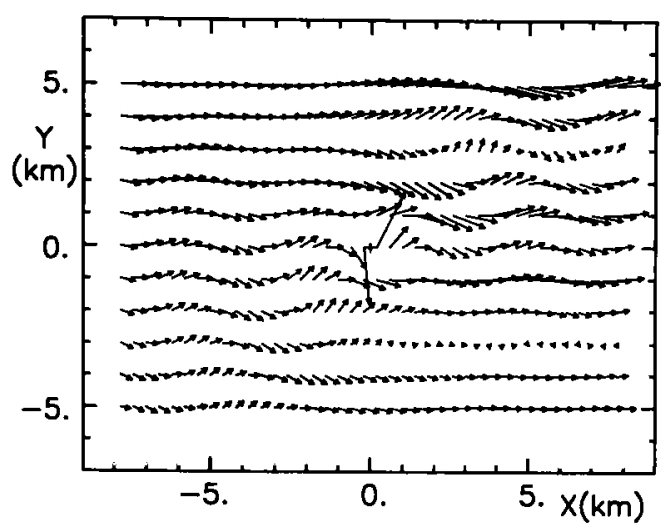

第 10 図流速べクトルの分布 $\left(U_{0}=0.02 \mathrm{~km} / \mathrm{s}, \kappa=\right.$ $0.1 / \mathrm{km}, \lambda=0.1 / \mathrm{km}, m=0.02 \mathrm{~km}^{2} / \mathrm{s}, \gamma=-0.02$ $\mathrm{km}^{2} / \mathrm{s}$ )

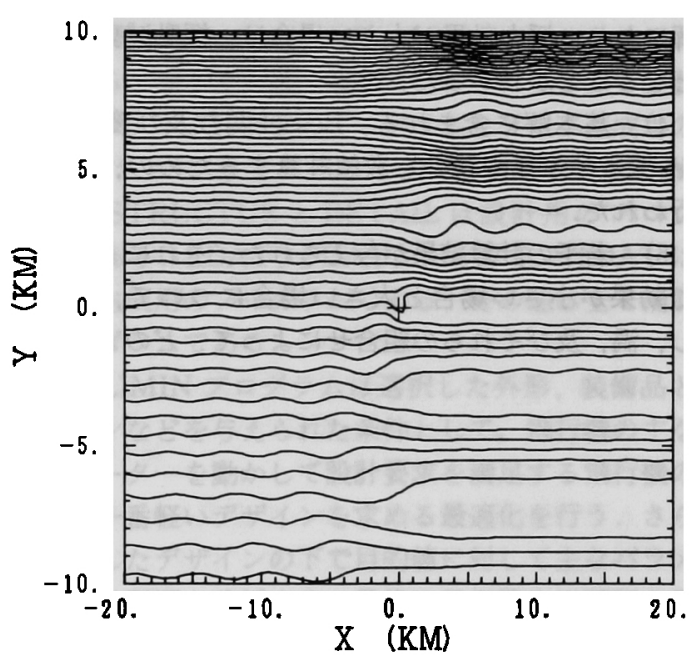

第 11 図流線図 $\left(U_{0}=0.02 \mathrm{~km} / \mathrm{s}, \kappa=0.1 / \mathrm{km}, \lambda=\right.$ $0.1 / \mathrm{km}, m=0.02 \mathrm{~km}^{2} / \mathrm{s}, \quad \gamma=-0.02 \mathrm{~km}^{2} / \mathrm{s}$ )

示したものである．流速の水平方向成分 $u$ も含めた 流れの振動的な様子が良くわかる.なおこの図におい ても，第 5 図と同じく流速の大きさは，目盛りに対し て， $x$ 方向に 25 倍， $y$ 方向に 100 倍されている.

第 11 図は，第 $9 ， 10$ 図と同じ条件のものを，流線 で示したものである. $x>0, y>0$ 及び $x<0, y<0$ の いずれの領域でも，流線は振動的になっているが， $x$ $>0, y>0$ の領域では, 特に $y$ 方向にある間隔をおい て，振幅がより大きくなる現象が現れていることがわ かる.

また，解が振動的となるこれらにおいて，yの絶対 值の大きいところで， $x$ に関する波長が長くなってい るが,これは，波長が特異点からの距離 $r$ によって 
いるからである（(42)式参照).

\section{5. 結}

論

高度が高くなるに従って，主流速度が指数関数的に 増加し，主流密度が指数関数的に減少する成層せん断 流について，流れの中に，吹き出し及び渦がおかれた ときの基本解の誘導を行った．得られた結論は以下の ようである。

1）渦及び吹き出しによる捻乱速度，擾乱圧力，嗄 乱渦度、擾乱流れ関数及び摄乱密度が得られた。

2）上記諸量の特異点近傍の特異性が明らかとなっ た，それらの特異性は，いずれも，一様流に対する特 異性に, 成層及びせん断流の効果を含んだ特異性が加 わった形をしていることがわかった。

3）特異点からある程度離れたところにおいては。 浮力効果が小さい場合は，単調に減衰するおだやかな 解になり．浮力効果が大きい場合は，振動減衰する解 となることがわかった。

4）基本解に含まれる，Bessel 関数及び変形 Bessel 関数の半無限積分を数値計算するための, 工夫が 行われた。

5）若干の計算結果が与えられた。それにより，浮 力効果が小さい場合と大きい場合について, 吹き出 し，渦，及びそれらの組合せによって，どのような流
れになるかが示された。

6）浮力効果が大きい場合，適当な吹き出しと洞の 重ね合わせによって, lee waveに似た波動を作り出 せることがわかった。

なお，本論の数值計算には，航空大学校のワークス テーション EWS 4800/50 (NEC) を用いた。要した計 算時間は，例えば，440点ての諸量を求めた第 10 図 の計算の場合, 約 1 分であった.

\section{参考文献}

1) 今井 功：流体力学(前編)，裳華房，東京，1973，pp.116119 .

2）柳沢三憲：境界要素法による全機形状の空力特性の計算， 日本航空宇宙学会誌，34 (1986)，pp.369-379.

3) Lighthill, M. J. : The Fundamental Solution for Small Steady Three-Dimensional Disturbances to a TwoDimensional Parallel Shear Flow, J. Fluid Mech., 3 (1957), pp. 113-144.

4) Ventres, C. S. : Shear Flow Aerodynamics Lifting Surface Theory, AIAA J., 13 (1975), pp. 1183-1189.

5）平野公孝，菊地正憲，佐藤秀坡：要音速せん断流中の定常 翼形特性，日本機械学会論文集 (B)，54 (1988). pp. 31753180

6) Turner, J.S.: Buoyancy Effects in Fluids, Cambridge University Press, Cambridge, 1973, pp. 34-37.

7) 森口繁一，宇田川銈久，一松 信：数学公式 I， II， III, 岩波蓄店，東京，1956，1957，1960。 